Please do not remove this page

RMIT

UNIVERSITY

\title{
Two output RF hybrid coupler using photonic transversal approach
}

Emami, Hossein; Sarkhosh, Niusha; Bui, Lam; Mitchell, Arnan

https://researchrepository.rmit.edu.au/esploro/outputs/9921863392501341/filesAndLinks?institution=61RMIT_INST\&index=null

Emami, H., Sarkhosh, N., Bui, L., \& Mitchell, A. (2007). Two output RF hybrid coupler using photonic transversal approach. 2007 Asia-Pacific Microwave Conference Proceedings, 1-4.

https://doi.org/10.1109/APMC.2007.4554701

Published Version: https://doi.org/10.1109/APMC.2007.4554701

Repository homepage: https://researchrepository.rmit.edu.au

(c) 2007 IEEE. Personal use of this material is permitted. However, permission to reprint/republish this material for advertising or promotional purposes or for creating new collective works for resale or redistribution to servers or lists, or to reuse any copyrighted component of this work in other works must be obtained from the IEEE.

Downloaded On 2023/04/27 00:55:56 +1000 


\title{
Two Output RF Hybrid Coupler Using Photonic Transversal Approach
}

\author{
Hossein Emami, Niusha Sarkhosh, Lam Anh Bui, and Arnan Mitchell \\ Microelectronics and Materials Technology Center \\ School of Electrical and Computer Engineering RMIT University \\ Melbourne, Australia \\ Email: $\underline{\text { Hossein.Emami@rmit.edu.au }}$
}

\begin{abstract}
A novel technique to implement a two output broad band RF hybrid coupler based on transversal signal processing is proposed and practically demonstrated. It features broadband frequency range, stable phase difference at outputs, $50 \mathrm{ohm}$ input/output impedance, and low noise characteristics. This technique is suitable for non-coherent optic implementation.
\end{abstract}

Keywords-component; transversal filter; microwave photonics, hybrid coupler;

\section{INTRODUCTION}

Quadrature hybrid couplers are important components for microwave circuits such as balanced mixers [1], beam formers $[2,3]$, and quadrature amplitude modulators [4]. These devices must provide low-loss; equal power splitting and maintain a precise $90^{\circ}$ phase shift over multiple octaves. Devices, realized using traditional microwave techniques [1-5], often have moderate bandwidth $(2-18 \mathrm{GHz})$ and good phase stability, but can exhibit significant amplitude imbalance due to a reliance on multi-resonant impedance matching. These devices can also have stringent fabrication tolerances [5] and poor port isolation [6-8].

In recent years, microwave photonics offers the possibility of ultra-broad bandwidths and excellent isolation and thus has been considered to improve quadrature hybrid implementations. Some solutions employ coherent optical beating to transfer the optical phase into the electrical domain $[9,10]$. Optical coherence must be maintained throughout these systems and this may be impractical in harsh defense environments.

Another microwave photonic technique that has been introduced recently is the photonic transversal filter [11-14]. This technique relies on time delays which are much less sensitive to vibration and temperature. We have recently proposed the use transversal filter to realize a broadband, highperformance quadrature hybrid coupler $[15,16]$. This paper presents an extension of this concept to two outputs and its practical implementation. Demonstrated performance is comparable to traditional microwave implementation. Paths for extension to ultra-broad bandwidths are discussed.

\section{THEORY OF OPERATION}

As discussed in [12-15], transversal filtering is a method by which a range of signal processing functions can be achieved. As shown in Fig. 1 a signal is divided between a number of taps with different weights $\left(w_{\mathbf{i}}, \mathrm{i}=1, \ldots, \mathrm{N}\right)$ and each tap experiences a different time delay $\left(t_{i}\right)$. At the output, all of the delayed taps are summed and detected. Each delayed tap in the transversal filter can be considered a distinct sample of a specific point in time. By adjusting the amplitude of each tap, an arbitrary impulse response can be formed. The frequency response of the summation of these delayed taps is the Fourier transform of the impulse response. Careful control of the tap amplitudes enables a broad range of filter functions to be realized.

It is well known that if a system has an impulse response of a homographic hyperbola type, the corresponding frequency response will have constant amplitude and a frequency independent $90^{\circ}$ phase shift relative to the input. The impulse response of such a Hyperbola is presented in Fig. 2 and the corresponding frequency response is presented in Fig. 3. For ideal system performance with infinite bandwidth, the impulse response must be an infinite continuous time signal. In reality, this is impractical, and thus the impulse response must be truncated in time. Windowing techniques are usually employed to reduce distortions of the system frequency response due to Gibes effect caused by abrupt truncation [15]. The impulse response must also be sampled by discrete taps which are effectively delta functions in time. It is worth mention that the sampling frequency must be at least as twice as the highest frequency component of the sampled signal to avoid aliasing

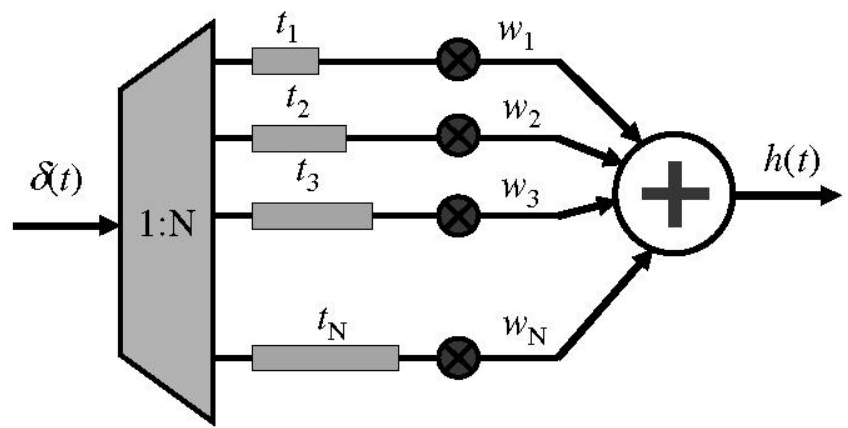

Fig. 1. Block diagram of transversal filter. 


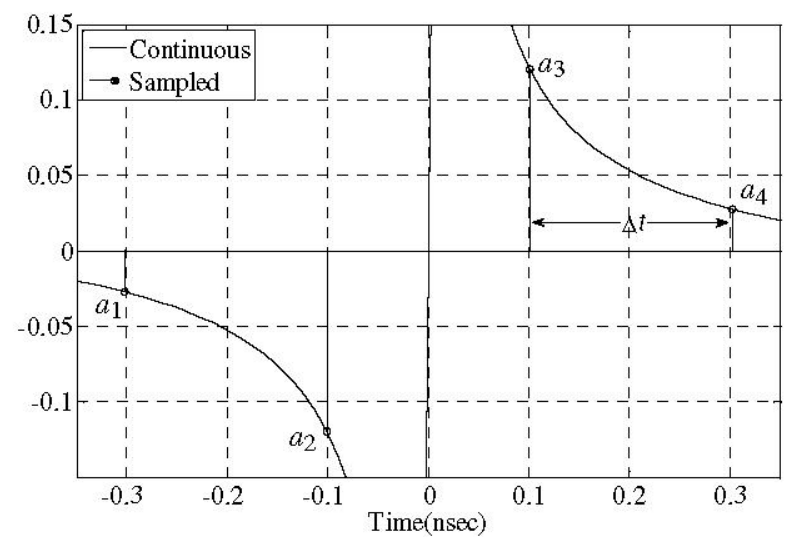

Fig. 2. Hyprebolic impulse response, continuous and infinite; and sampled.

The impulse response in transversal filter is sampled by discrete taps which are effectively delta functions in time. Thus, along with being truncated, the impulse response is samples as shown in Fig. 2. Ideally, appropriate windowing should enable synthesis of a practical filter with performance similar to its continuous counterpart. Fig. 3 presents the predicted amplitude and phase of the frequency response of both the continuous, infinite and also the sampled and truncated responses shown in Fig. 2. As it can be seen, sampling hasn't affected the phase.

\section{METHOD}

To implement the transversal filter depicted in Figs. 2 and 3, a Wavelength Division Multiplexing (WDM) photonic technique has been chosen. To demonstrate the concept of photonic RF hybrid coupler four lasers with different wavelength have been used to implement a four taps transversal filter using wavelength division multiplexing
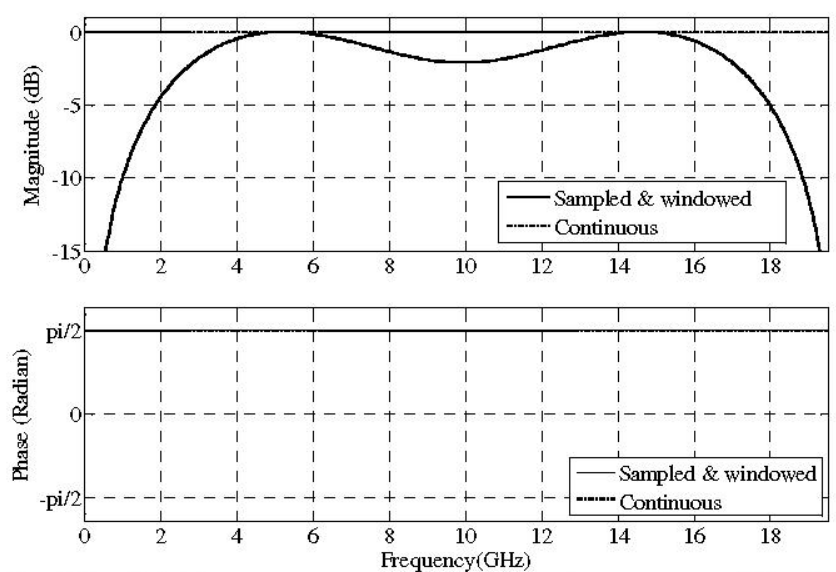

Fig. 3.Predicted frequency response for the impulse responses of Fig. 1: continuous and sampled.

(WDM). Fig. 4 presents a diagram of the system implementation. Two sets of lasers are combined and input to two oppositely biased Mach-Zehnder Modulators to achieve positive $\left(\mathrm{V}^{+}\right)$and negative $\left(\mathrm{V}^{-}\right)$taps. Polarization Controllers on each wavelength ensure effective modulation. The path lengths were equalized after each modulator using variable optical lengths. The taps were then combined and a dispersive fiber was used to impart different time delays to each wavelength. The taps were amplified using an erbium doped fiber amplifier and then a portion of one tap was separated using a wavelength division multiplexer coupler. The path lengths were again equalized with variable optical lengths before detecting using broadband photo-detectors. The multiwavelength output (Out1) formed the $90^{\circ}$ phase shifted signal, while the single wavelength output (Out2) provided a $0^{\circ}$ reference. The output frequency response was observed with a vector network analyzer.

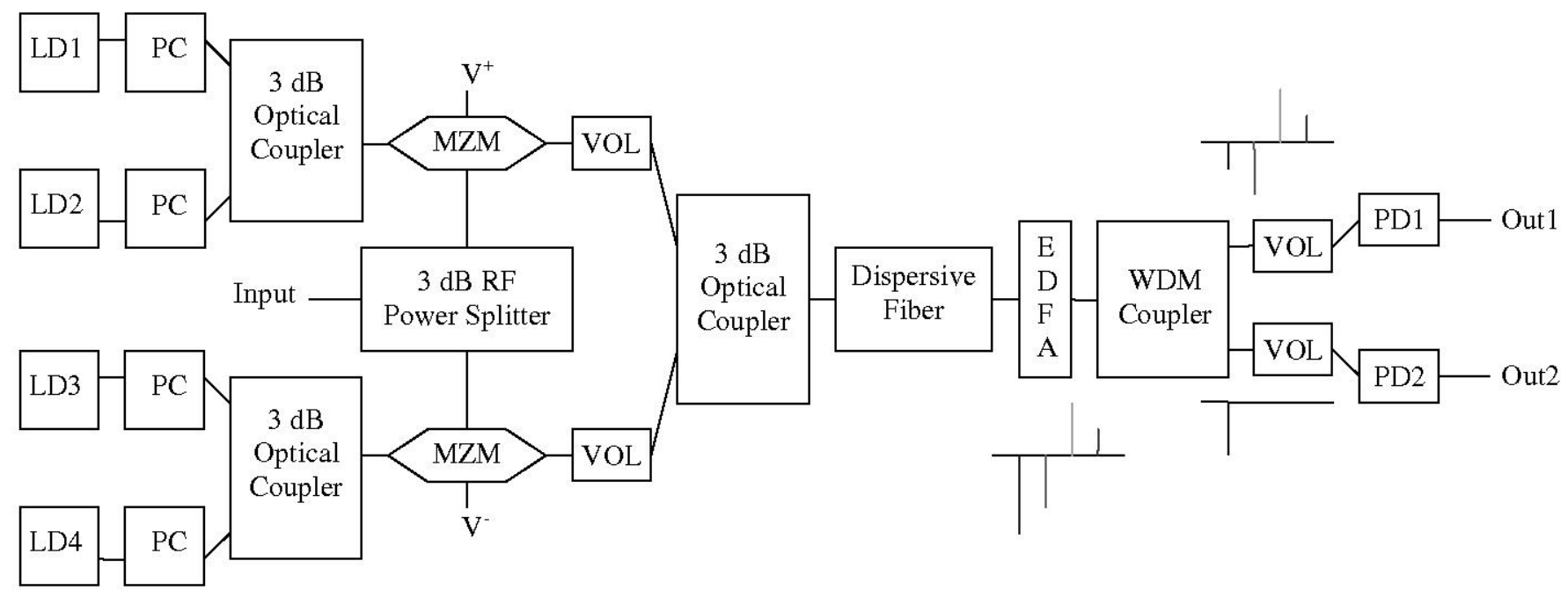

Fig. 4. Amplitude of the frequency response of the transversal filter. LD=Laser Diode; $\mathrm{PC}=$ Polarisation Controller; $\mathrm{MZM}=\mathrm{Mach}-\mathrm{Zehnder} \mathrm{Modulator}$; VOL=Variable Optical Lengths; EDFA = Erbium Doped Fiber Amplifier; WDM=Wavelength Division Multiplexer; PD= Photodetector. 


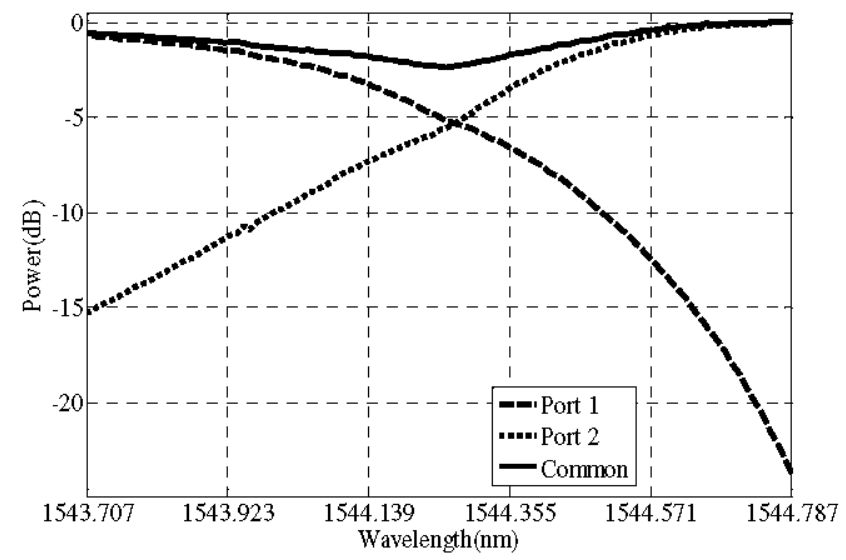

Fig. 5. Wavelength division mutliplexer charactristic

The power of each laser determines the weight of each tap. Due to the limited number of available lasers, our implementation used 4 taps. As discussed in $[15,16]$, to achieve a $20 \mathrm{GHz}$ bandwidth, the time spacing of the samples must be $\Delta t \approx 50 \mathrm{ps}$. This delay was achieved using $2.354 \mathrm{~km}$ of single mode fiber with dispersion $\mathrm{D}=17 \mathrm{ps} /(\mathrm{nm} \cdot \mathrm{km})$. As:

$$
\Delta \lambda=\Delta \mathrm{t} / \mathrm{DL}
$$

the time spacing and fiber length were used to calculate the wavelength spacing as: $\Delta \lambda=1.25 \mathrm{~nm}$. Since the dispersion was not exactly linear and non-trivial dispersion was contributed by other system components, the actual wavelength of the lasers was empirically fine-tuned to achieve the required time delay. This was achieved by isolating pairs of anti-symmetric taps and adjusting their wavelength spacing to ensure a null in the frequency response at $20 \mathrm{GHz}$. The resulting wavelength taps were $1543.94,1545.3,1546.59$ and $1547.69 \mathrm{~nm}$.

Fig. 5 presents the response of the 3-port wavelength division multiplexer. High and low wavelengths are routed to Ports 1 and 2 respectively, with a gradual transition across $1544.2 \mathrm{~nm}$. A wavelength of $1543.94 \mathrm{~nm}$ was chosen for the lowest tap such that most of the power was isolated at Port 2 to act as a reference, while a portion remained at Port 1 to contributed to the transversal filter. Only one wavelength will

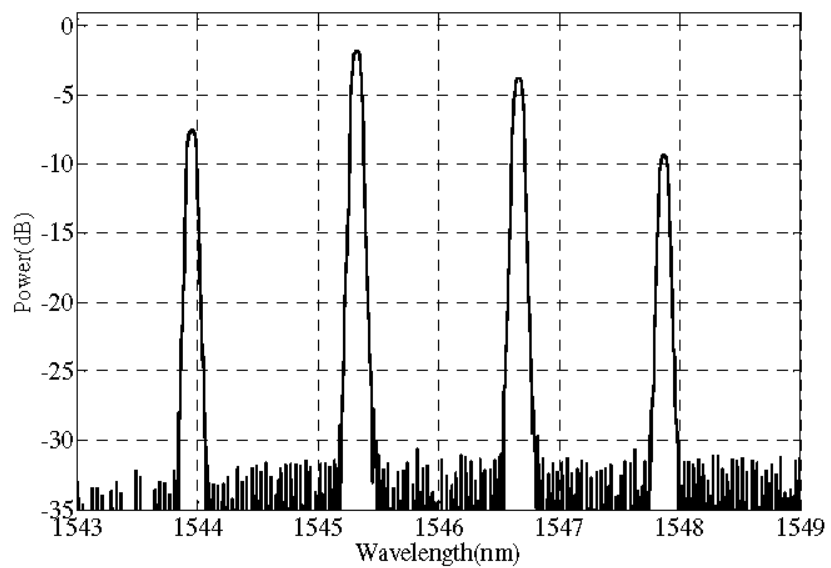

Fig. 6. Optical Spectrum Analyser trace showing weights and wavelength of each tap.
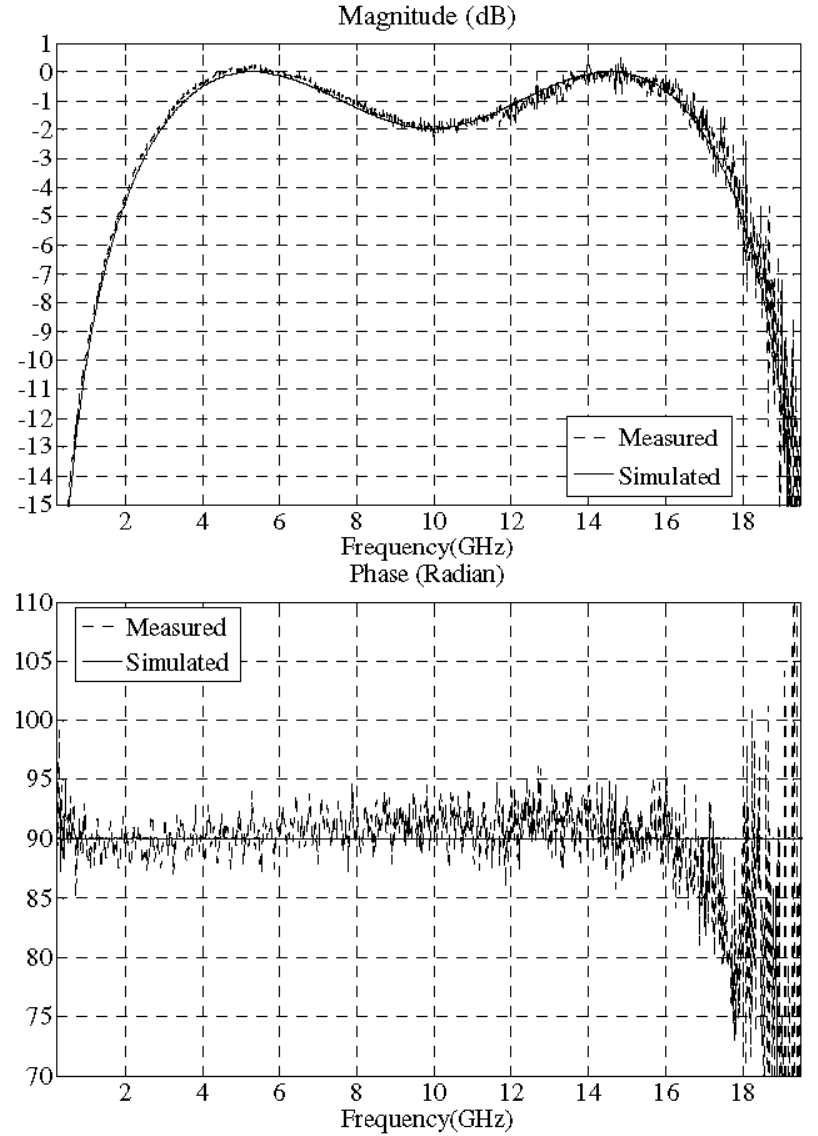

Fig. 7. Measured and predicted system magnitude and phase response

thus be required for both functions.

\section{RESULTS}

The system was configured as shown in Fig. 4. The laser powers were adjusted to achieve the tap weights indicated in Fig. 2. Fine tuning was achieved by observing the frequency response of anti-symmetric pairs of taps in isolation and adjusting their separation and relative weights to achieve a deep amplitude null at $20 \mathrm{GHz}$. The relative amplitudes and wavelength spacing of the two pairs of taps was then adjusted to achieve the correct phase and amplitude response. Fig. 6 presents the optical spectrum analyzer trace indicating the relative wavelengths and amplitudes of the transversal filter.

The vector network analyzer was calibrated to the reference arm (Out2) and then used to measure the frequency response of Out1. The amplitude and phase response of the transversal filter (Out1) is presented in Figs. 7. The simulated response of Fig. 2 is also presented for comparison. Excellent agreement between the measurement and theory is evident. Amplitude variation $<2 \mathrm{~dB}$ has been maintained over $2.9-17 \mathrm{GHz}$, with less than $5^{\circ}$ phase deviation from $90^{\circ}$.

\section{DISCUSSION}

The tap weights depicted in Fig. 6 do not appear to have equal values, despite being empirically optimized because the 
tap weights are determined by the power of each wavelength, not the peak intensity. In a practical implementation, the optimization of channels could be achieved using real time monitoring and numerical control as there are only a small number of variables and clear optimization goals. The phase degradation in Fig. 7 above $16 \mathrm{GHz}$ could be attributed to the bandwidth limitation of the $3 \mathrm{~dB}$ RF splitter at the modulator inputs. A single balanced modulator could replace the two opposite biased modulators, eliminating the need for an RF splitter. This would also simplify path length equalization; reduce component count and optical splitting losses. Replacing the $3 \mathrm{~dB}$ couplers with wavelength division multiplexers and use of polarization maintaining components will further simplify the system implementation.

The response of Fig. 7 compares favorably traditional microwave hybrids. This photonic implementation offers the flexibility of a customized pass band. The pass band ripple and the bandwidth can be dynamically adjusted by changing the tap weights and wavelength spacing. It would be desirable to extend the hybrid operation to $2-40 \mathrm{GHz}$ with less than $3 \mathrm{~dB}$ ripple. To improve the bandwidth and ripple, extra taps could be introduced as discussed in $[15,16]$. Systems with these suggested improvements are currently under investigation.

\section{CONCLUSION}

A novel two output quadrature hybrid coupler has been implemented using a photonic transversal scheme. This implementation offers dynamically adjustable bandwidth and ripple. The isolation between outputs is excellent without requiring impedance matching. Extension to broader bandwidth through introduction of extra wavelength channel taps is possible.

\section{REFERENCES}

[1] M. Aikawa, and H. Oqawa, "Double-sided MICs and their applications," EEE Trans. MTT, vol. 37, pp. 406-413, Feb. 1989.

[2] P. Slonen, P. Jokinen, M. Keskilammi, and L. Sydänheimo, "Analysis of $2.45 \mathrm{GHz} 4$-Bit hybrid-coupled phase shifter for phased arrays," in Proc.
IEEE Region 10 Int. Conf. Electrical and Electronic Tech., Phuket Island, Langkawi Island, Singapore, vol.2, pp. 529-534, Aug. 2001.

[3] R. T. Chen, E. R. Brown, R. S. Singh, "A compact $30 \mathrm{GHz}$ low loss balanced hybrid coupler fabricated using micromachined integrated coax," in 2004 IEEE Radio and Wireless Conf., Atlanta, GA/USA, pp. 227-230, Sep. 2004,.

[4] S. Kumar, A. Mohammadi and D. Klymyshyn, "A direct 64QAM modulator suitable for MMIC applications," Microwave Joumal, vol.40, pp. 116-122, Apr. 1997.

[5] L. Fan, b. Heimer, and K. Chang, "Uniplanar hybrid couplers using asymmetrical coplanar strip lines," IEEE MTT-S Int. Microwave Symp. Dig., Dever, CO/USA, vol. 1,pp. 273-276, Jun. 1997.

[6] M. -H. Murgulescu, P. Leguad, E. Penard, and I. Zaquine, "New small 90 degree hybrid coupler," Electron. Lett., vol. 30, pp. 1289-1290, Aug. 1994.

[7] C. H. Ho, L. Fan, and K. Chang, "Broad-band Uniplanar hybrid-ring and branch-line couplers," IEEE Trans. MTT, vol. 41, pp. 2116-2125, Dec. 1993.

[8] B. R. Heimer, "Uniplanar hybrid couplers using asymmetrical coplanar striplines," IEEE Trans. MTT, vol. 45, pp. 2234-2240, Dec 1997.

[9] G. A. Berenbrock and B. Schelmmer, "Active controlled fiber optical 90 degree hybrid for coherent communications," EEE Photon. Tech. Lett., vol.1, pp. 86-87, Apr. 1989.

[10] D. Madris, B. Vidal, A. Martinez, V. Polo, J. L. Corral, and J. Marti, “A novel $2 \mathrm{~N}$ beams heterodyne optical beamforming architecture based on N×N optical butler matrices," IEEE MTT-S International Microwave Symposium Digest, Seattle, WA/USA, vol.3 pp. 1945-1948, Jun. 2002.

[11] L. V. T. Nguyen, G. Edvell, D. B. Hunter, and M. Englund, "Wideband in-phase and quadrature microwave signal generation utilizing a multichannel chirped fiber grating," in Proc Australian Conf. on Optical fiber Tech., Melbourne, VIC/Australia, pp. 25-27, Jul. 2006.

[12] J. Capmany, J. Mora, D. Pastor, and B. Ortega, "High-quality onlinereconfigurable microwave photonic transversal filter with positive and negative coefficients," EEE Photon. Tech. Lett., vol.17, pp. 2730-2732, Dec. 2005.

[13] S. Mansoori, A. Mitchell, K. Ghorbani "Photonic reconfigurable microwave filter with negative coefficients" Electron. Lett. , vol. 40, pp 541-543, Apr. 2004.

[14] S. Mansoori, A. Mitchell, "RF transversal filter using an AOTF," EEEE. Photon. Tech. Lett., vol. 16, pp 879-881, Mar. 2004.

[15] H. Emami, L. A. Bui, A. Mitchell, "Broadband 90 degree hybrid coupler using photonic transversal approach," in Proc. Asia-Pacific Microwave Photon. Conf., Kobe, Japan, pp. 173-176, Apr. 2006.

[16] H. Emami, L. A. Bui, S. Mansoori, A. Mitchell, "Quadrature hybrid coupler using photonic transversal approach," in Australia Conf. on Optical Fiber Tech., Melbourne, VIC/Australia, D: Paper 4, Jul. 2006. 